\title{
Estudo comparativo entre rivaroxaban e enoxaparina na profilaxia de tromboembolismo venoso profundo em pacientes submetidos à artroplastia total do quadril
}

\author{
Comparative study between rivaroxaban and enoxaparin \\ in deep venous thromboembolism prophylaxis in \\ patients submitted to total hip arthroplasty
}

Pedro Silva Kanan ${ }^{1}$, Carlos Roberto Schwartsmann², Leonardo Carbonera Boschin ${ }^{3}$, Samuel Conrad ${ }^{4}$, Marcelo Faria Silva ${ }^{5}$

\section{RESUMO}

Objetivo: Comparar a eficácia e segurança de rivaroxaban com a enoxaparina na profilaxia de trombose venosa profunda (TVP) após artroplastia total de quadril. Métodos: No período de setembro de 2006 a abril de 2007, no Serviço de Ortopedia e Traumatologia do Complexo Hospitalar da Santa Casa de Porto Alegre/RS, foi realizado um ensaio clínico randomizado, duplo-cego, em que foram

* Trabalho realizado no Serviço de Ortopedia e Traumatologia do *Complexo Hospitalar Santa Casa de Porto Alegre/RS/Brasil (ISCMPA/RS).

1. Residente do $3^{\circ}$ ano do Serviço de Ortopedia e Traumatologia do Complexo Hospitalar Santa Casa de Porto Alegre (RS), Brasil.

2. Doutor, Professor Titular de Ortopedia da Universidade Federal de Ciências da Saúde de Porto Alegre - UFCSPA - Porto Alegre (RS), Brasil.

3. Médico do Grupo do Quadril do Complexo Hospitalar Santa Casa de Porto Alegre, Porto Alegre (RS), Brasil.

4. Acadêmico do 6c ano do Curso de Graduação da Universidade Federal de Ciências da Saúde de Porto Alegre - UFCSPA - Porto Alegre (RS), Brasil. Estagiário do Serviço de Ortopedia e Traumatologia ISCMPA/RS.

5. Mestre, Professor do Curso de Fisioterapia do Centro Universitário Metodista - IPA - Porto Alegre (RS), Brasil.

Endereço para correspondência: Prof. Dr. Carlos Roberto Schwartsmann, Rua Leopoldo Bier, 825, Bairro Santana - 90620-100 - Porto Alegre (RS), Brasil. Tel.: (51) 3214-8074 (manhã), (51) 32176422 (tarde). E-mail: schwartsmann@santacasa.tche.br

Recebido em 30/5/08. Aprovado para publicação em 30/7/08.

Copyright RBO2008 selecionados 67 pacientes, todos submetidos à artroplastia total de quadril (ATQ). Desses, foram excluídos dois pacientes por falta de adesão à profilaxia proposta após a alta hospitalar. Para um dos grupos foi administrada enoxaparina $40 \mathrm{mg}$, subcutânea seis a oito horas antes da cirurgia e, depois desta, foi adicionado um comprimido de placebo, via oral, durante os primeiros 32 a 36 dias, uma vez ao dia. $O$ outro grupo recebeu rivaroxaban $10 \mathrm{mg}$, via oral, uma vez ao dia, durante os primeiros 32 a 36 dias pós-operatórios. Para fazer o cegamento dos grupos foi administrada uma injeção de placebo subcutâneo seis a oito horas antes da cirurgia e nos 32 a 36 dias subseqüientes. $O$ desfecho principal estudado foi a eficácia na prevenção de TVP, que foi avaliada através de venografia bilateral realizada entre os dias 32 e 36 de pós-operatório e/ou através de sintomas documentados de trombose venosa profunda ou tromboembolismo pulmonar (TEP). $O$ desfecho secundário estudado foi segurança na posologia, avaliada através de sangramento importante e/ou hepatotoxicidade. Resultados: Rivaroxaban e enoxaparina tiveram resultados semelhantes (as diferenças não foram estatisticamente significativas), quando comparados quanto à redução da incidência de TVP até o $36^{\circ}$ dia pós-operatório. Não houve diferença quanto à perda sanguínea e a hepatotoxicidade, quando comparadas as drogas. Conclu- 
são: Em pacientes submetidos à artroplastia total de quadril, o rivaroxaban mostrou-se igualmente eficaz e seguro quanto a enoxaparina na prevenção de trombose venosa profunda e tromboembolismo pulmonar.

\footnotetext{
Descritores - Trombose venosa/prevenção \& controle; Embolia pulmonar/prevenção \& controle; Artroplastia de quadril; Enoxaparina; Heparina; Estudo comparativo
}

\section{ABSTRACT}

Objective: To compare the effectiveness and safety of rivaroxaban to those of enoxaparin for the prophylaxis of deep venous thrombosis (DVT) after total hip arthroplasty. Methods: From September 2006 to April 2007, at the Orthopedics and Traumatology Clinic of the Hospital Complex of the Santa Casa of Porto Alegre, State of Rio Grande do Sul, a randomized, double-blind clinical trial was carried out in which 67 patients were selected $(n=$ 67), all of them submitted to total hip arthroplasty (ATQ). Of these patients, two were excluded for lack of adherence to the prophylaxis proposed after hospital release $(n=65)$. One of the groups was given subcutaneous $40 \mathrm{mg}$ enoxaparin 6 hours to 8 hours before surgery, and after surgery a placebo pill was added, for once a day oral intake, during the first 32 to 36 days. The other group was given oral $10 \mathrm{mg}$ rivaroxaban, once a day, during the first 32 to 36 post-operative days. In order to have the double-blind feature of the study, a subcutaneous placebo injection was given 6 hours to 8 hours before surgery and on the 32 to 36 days following surgery. The main outcome aimed at was the effectiveness in preventing DVT, which was evaluated by bilateral venography carried out between days 32 and 36 after surgery, or through documented symptoms of deep venous thrombosis or pulmonary thromboembolism (PTE). The secondary outcome studied was administration safety, that was evaluated through major bleeding or liver toxicity. Results: Rivaroxaban and enoxaparin showed similar results (the differences were not statistically significant) when compared for the reduction of DVT incidence till the $36^{\text {th }}$ post-operative day. There was no difference in blood loss and liver toxicity when the drugs were compared. Conclusion: In patients submitted to total hip arthroplasty, rivaroxaban showed as effective and safe as enoxaparin to prevent deep venous thrombosis and pulmonary thromboembolism.

\section{Keywords - Venous thrombosis/prevention \& control; Pulmonary embolism/prevention \& control; Arthroplasty, replacement, hip; Enoxaparin; Heparin; Comparative study}

\section{INTRODUÇÃO}

A artroplastia total de quadril (ATQ) é considerada um dos procedimentos cirúrgicos mais bem sucedidos da ortopedia. Proporciona alívio da dor e recuperação da mobilidade da articulação do quadril, melhorando sensivelmente a qualidade de vida do paciente.

Apesar dos benefícios indiscutíveis do procedimento, complicações locais e sistêmicas podem ocorrer. As complicações locais mais citadas são: lesões vasculonervosas, luxações da prótese, fraturas do fêmur e infecção ${ }^{(1)}$.

Fenômenos tromboembólicos representam a principal complicação sistêmica de $\mathrm{ATQ}^{(2-3)}$. Trombose venosa profunda (TVP) ocorre em aproximadamente 40$70 \%$ dos pacientes submetidos à artroplastia dos membros inferiores que não fazem uso de medidas profiláticas $^{(2-7)}$. Pequeno número de pacientes, entre 1 e 3\%, que desenvolve TVP irá evoluir para tromboembolia pulmonar (TEP), que é a maior causa de morte nos três primeiros meses após a operação, representando mais de $50 \%$ da mortalidade pós-operatória ${ }^{(5,7-8)}$.

Inúmeros estudos têm sido feitos na busca de um método eficaz e seguro na profilaxia de eventos tromboembólicos nas cirurgias de grande porte, como é o caso da ATQ. O surgimento de métodos cientificamente eficazes para esse propósito propiciou diminuição significativa do risco cirúrgico e dos custos hospitalares e, conseqüentemente, maior segurança para o paciente e para o cirurgião. No entanto, ainda não há consenso na literatura sobre qual o mais eficiente fármaco a ser indicado para a profilaxia dos fenômenos tromboembólicos associados à ATQ. 
O objetivo deste trabalho é avaliar a eficácia e segurança de rivaroxaban $10 \mathrm{mg}$ por dia, via oral, na profilaxia do tromboembolismo venoso em pacientes submetidos à ATQ, quando comparado com a enoxaparina 40mg, via subcutânea, uma vez ao dia.

\section{MÉTODOS}

No período de setembro de 2006 a abril de 2007, no Serviço de Ortopedia e Traumatologia do Complexo Hospitalar Santa Casa de Porto Alegre, foi realizado ensaio clínico randomizado, duplo-cego. Selecionaram-se 67 pacientes, os quais foram submetidos à artroplastia total de quadril (ATQ). Para um dos grupos foi administrada enoxaparina, 40mg, por via subcutânea de seis a oito horas antes da cirurgia e, depois desta, foi adicionado um comprimido de placebo, por via oral, uma vez ao dia, durante os primeiros 32 a 36 dias (grupo ENO). Outro grupo recebeu rivaroxaban 10mg, por via oral, uma vez ao dia, durante os primeiros 32 a 36 dias de pós-operatórios. O mascaramento deste grupo foi feito pela administração de uma injeção de placebo via subcutânea seis a oito horas antes da cirurgia e nos 32 a 36 dias subsequientes (grupo RIV). Durante a evolução do estudo, dois pacientes foram excluídos devido à não adesão à profilaxia proposta após a alta hospitalar. Com isso, a população efetiva do estudo foi de 65 pacientes, sendo 33 no grupo RIV e 32 no grupo ENO.

Dados demográficos - Dentre os pacientes selecionados para o estudo, houve predominância do sexo masculino, tanto no grupo RIV quanto no grupo ENO (55\% e $56 \%$, respectivamente), cor branca ( $88 \%$ e 94\%) (tabela 1). Dados clínicos - Os dados clínicos analisados foram idade, peso, altura, índice de massa corporal (IMC), pressão arterial sistólica (PAS), pressão arterial diastólica (PAD) e freqüência cardíaca (FC). A média de idade foi de 54,5 anos no grupo RIV e 61,3 anos no grupo ENO. O paciente mais jovem do estudo tinha 20 anos e o mais velho, 82 anos (tabela 2). Inclusão de pacientes - Foram selecionados para o presente estudo pacientes que atendiam aos seguintes critérios de inclusão: a) 18 anos de idade ou mais; b) prótese de quadril com indicação de cirurgia eletiva; c) pacientes que assinaram termo de consentimento.
TABELA 1

Caracterização da amostra

\begin{tabular}{|c|c|c|c|}
\hline & & $\begin{array}{c}\text { Grupo rivaroxaban } \\
\text { (n = 33) } \\
n(\%)\end{array}$ & $\begin{array}{c}\text { Grupo enoxaparina } \\
(n=32) \\
n(\%)\end{array}$ \\
\hline Sexo & $\begin{array}{l}\text { Masculino } \\
\text { Feminino }\end{array}$ & $\begin{array}{l}18(55) \\
15(45)\end{array}$ & $\begin{array}{l}18(56) \\
14(44)\end{array}$ \\
\hline Cor & $\begin{array}{l}\text { Branco } \\
\text { Negro }\end{array}$ & $\begin{array}{r}29(88) \\
4(12)\end{array}$ & $\begin{array}{rr}30 & (94) \\
2 & (6)\end{array}$ \\
\hline
\end{tabular}

Fonte: CEDOP - Complexo Hospitalar Santa Casa de Porto Alegre * Valor $\mathrm{p}<0,05$

TABELA 2

Descrição do perfil demográfico

\begin{tabular}{|c|c|c|}
\hline & $\begin{array}{c}\text { Grupo } \\
\text { rivaroxaban } \\
(\mathrm{n}=33) \\
\bar{X}(\mathrm{DP})\end{array}$ & $\begin{array}{c}\text { Grupo } \\
\text { enoxaparina } \\
\text { (n }=32) \\
\bar{X} \text { (DP) }\end{array}$ \\
\hline Idade (anos) & $54,5(13,9)$ & $61,3(12,8)$ \\
\hline Peso $(\mathrm{kg})$ & $68,8(13,9)$ & $76,1(15,3)$ \\
\hline Estatura (metros) & $1,63(0,1)$ & $1,65(0,1)$ \\
\hline $\mathrm{IMC}\left(\mathrm{kg} / \mathrm{m}^{2}\right)$ & $25,5 \quad(4,3)$ & $27,6 \quad(5,5)$ \\
\hline Pressão arterial sistólica (mmHg) & $130,1(15,5)$ & $133,2(17,0)$ \\
\hline Pressão arterial diastólica $(\mathrm{mmHg})$ & $80,5(10,5)$ & $82,4(10,6)$ \\
\hline Freqüência cardíaca (bpm) & $73,3 \quad(9,7)$ & $73,4(10,0)$ \\
\hline
\end{tabular}

Fonte: CEDOP - Complexo Hospitalar Santa Casa de Porto Alegre * Valor $\mathrm{p}<0,05$

Exclusão de pacientes - Não participaram do estudo pacientes que se enquadravam em pelo menos um dos seguintes critérios: a) programados para cirurgia eletiva de prótese de quadril bilateral; b) com sangramento ativo ou alto risco de sangramento, contra-indicando o tratamento com heparina de baixo peso molecular; c) portadores de contra-indicação relacionada à droga a ser usada ou condição que impedisse o tratamento com anticoagulante; d) portadores de condição que contra-indicasse a realização de flebografia bilateral; e) grávida ou lactante; f) mulheres em idade fértil que não estivessem utilizando métodos anticoncepcionais adequados; g) viciados em drogas ou álcool; h) em uso concomitante de inibidores da protease do HIV; i) em vigência de terapia com outro produto em estudo dentro do período de 30 dias antes do início da pesquisa clínica; j) em uso de compressão pneumática intermitente durante o período de tratamento ativo; k) submetidos concomitantemente a outra pesquisa clínica ou estudo; 1) em terapia com outro tipo de anticoagu- 
lante que não pode ser interrompida ou outra medicação não permitida; m) doença hepática importante. Dados laboratoriais pré-operatórios - Foram avaliados no pré-operatório os seguintes exames laboratoriais: hemograma completo, sódio, potássio, creatinina, glicemia em jejum, TGO, TGP, fosfatase alcalina, bilirrubinas total e direta, lipase, amilase e desidrogenase lática (tabela 3). Diagnóstico pré-operatório Foram considerados os seguintes diagnósticos préoperatórios entre os pacientes do estudo: osteoartrite do quadril, necrose avascular da cabeça do fêmur, seqüela de fratura do colo do fêmur, displasia do desenvolvimento do quadril, espondilite anquilosante, artrite reumatóide e outros. Osteoartrite do quadril foi o diagnóstico mais prevalente, sendo encontrada em 17 pacientes no grupo RIV e em 22 no grupo ENO (tabela 4). Fatores de risco - Os fatores de risco considerados para a análise em questão foram: obesidade, tendo como base o cálculo de índice de massa corporal (IMC) maior que $30 \mathrm{~kg} / \mathrm{cm}^{2}$ e tabagismo (tabela 5 ).

Antes da cirurgia os pacientes foram avaliados clínica e laboratorialmente e submetidos à triagem dos critérios de inclusão e exclusão. Além disso, foram esclarecidos sobre os propósitos e implicações do estudo; após todos terem assinado o termo de consenti-

TABELA 3

Descrição dos exames laboratoriais no pré-operatório

\begin{tabular}{|c|c|c|c|}
\hline & $\begin{array}{c}\text { Grupo } \\
\text { rivaroxaban } \\
(\mathbf{n}=33) \\
\bar{X}(D P)\end{array}$ & $\begin{array}{c}\text { Grupo } \\
\text { enoxaparina } \\
\text { (n = 32) } \\
\bar{X}(D P)\end{array}$ & $\begin{array}{l}\text { Valor } \\
\text { de p }\end{array}$ \\
\hline Hemoglobina (g/dL) & $13,4 \quad(1,3)$ & $13,5 \quad(1,6)$ & 0,413 \\
\hline Hematócrito (\%) & 38,6 & $(4,5)$ & \\
\hline $\operatorname{citos}\left(\times 10^{3} / \mathrm{mm}^{3}\right)$ & $8,0 \quad(3,0)$ & $7,0 \quad(2,0)$ & \\
\hline $\mathrm{s}\left(\mathrm{x} 10^{3} / \mathrm{mm}^{3}\right)$ & $261 \quad(70,0)$ & $247 \quad(60,0)$ & 0,198 \\
\hline Sódi & $140,8 \quad(4,8)$ & $139,7 \quad(2,1)$ & 0,129 \\
\hline Pot & $4,1 \quad(0,4)$ & $4,1 \quad(0,3)$ & 0,451 \\
\hline & $1,0 \quad 10$ & $1,1 \quad(0,3)$ & 0,0 \\
\hline $\mathrm{g} / \mathrm{dL})$ & $86,6(12$, & $95,1(33,9)$ & \\
\hline & $20,3 \quad(7,3$ & $19,3 \quad(4,4)$ & 0,257 \\
\hline TGP $(U / L)$ & $22,4 \quad(7,3)$ & $22,1(13,1)$ & 0,449 \\
\hline GGT (U/L) & $58,0(23,9)$ & $30,4(24,1)$ & 0,495 \\
\hline Bilirrubina total (mg/dL) & $0,4 \quad(0,2)$ & $0,3 \quad(0,2)$ & 0,226 \\
\hline Bilirrubina direta $(\mathrm{mg} / \mathrm{dL})$ & $0,2 \quad(0,2)$ & $0,2 \quad(0,1)$ & 0,196 \\
\hline Fosfatase alcalina (U/L) & $83,5(28,9)$ & $76,1(21,4)$ & 0,122 \\
\hline
\end{tabular}

Fonte: CEDOP - Complexo Hospitalar Santa Casa de Porto Alegre

Rev Bras Ortop. 2008;43(8):319-28 mento informado, foram incluídos e randomizados em dois grupos.

Os pacientes foram internados um dia antes da cirurgia: dia 0 do estudo, para serem preparados. Durante esse dia foram coletadas amostras de sangue para exames laboratoriais, realizados eletrocardiograma e radiografia de tórax, para avaliação pré-operatória. Entre seis e oito horas antes da cirurgia foi administrada uma ampola por via subcutânea; no grupo ENO, continha 40mg de enoxaparina e, no grupo RIV, placebo.

No dia seguinte, os pacientes foram submetidos à artroplastia total de quadril, considerado o dia 1. A via de acesso ântero-lateral foi a mais utilizada em ambos os grupos, sendo $82 \%$ no grupo RIV e $72 \%$ no grupo ENO. No grupo RIV o lado mais operado foi o direito (58\%), enquanto no grupo ENO, o lado esquerdo (59\%). Em ambos os grupos, a prótese cimentada foi a mais utilizada, sendo $70 \%$ no grupo RIV e $72 \%$ no grupo ENO; o tipo de anestesia mais realizada foi a raquidiana, sendo $64 \%$ no grupo RIV e $56 \%$ no grupo ENO. O

TABELA 4

Valores de ocorrência do diagnóstico no pré-operatório

\begin{tabular}{|c|c|c|}
\hline & $\begin{array}{c}\text { Grupo } \\
\text { rivaroxaban } \\
\text { (n = 33) } \\
n(\%)\end{array}$ & $\begin{array}{c}\text { Grupo } \\
\text { enoxaparina } \\
(n=32) \\
n(\%)\end{array}$ \\
\hline Osteoartrite & $17(52)$ & $22(69)$ \\
\hline Necrose avascular & $8(24)$ & $3(9)$ \\
\hline Seqüela de fratura de colo de fêmur & $2(6)$ & $3(9)$ \\
\hline Displasia do desenvolvimento do quadril & $2(6)$ & $2(7)$ \\
\hline Espondilite anquilosante & $2(6)$ & $1(3)$ \\
\hline Artrite reumatóide & $1(3)$ & $1(3)$ \\
\hline Outros & $1(3)$ & $0 \quad(-)$ \\
\hline \multicolumn{3}{|c|}{$\begin{array}{l}\text { Fonte: CEDOP - Complexo Hospitalar Santa Casa de Porto Alegre } \\
\text { * Valor } p<0,05\end{array}$} \\
\hline \multicolumn{3}{|l|}{ TABELA 5} \\
\hline \multicolumn{3}{|c|}{ Valores de ocorrência de fatores de risco no pré-operatório } \\
\hline $\begin{array}{c}\text { Grupo rivaroxaban } \\
\text { (n = 33) } \\
n(\%)\end{array}$ & \multicolumn{2}{|c|}{$\begin{array}{c}\text { Grupo enoxaparina } \\
\text { (n = 32) } \\
n(\%)\end{array}$} \\
\hline Obesidade & \multicolumn{2}{|c|}{$7(22)$} \\
\hline Tabagismo & \multicolumn{2}{|c|}{$15(47)$} \\
\hline
\end{tabular}

Fonte: CEDOP - Complexo Hospitalar Santa Casa de Porto Alegre * Valor $\mathrm{p}<0,05$ 
tempo de cirurgia variou entre 55 e 170 minutos no grupo RIV, e 65 e 200 minutos no grupo ENO, com tempo médio de 91,8 minutos e 102,9 minutos, respectivamente. O sangramento médio no transoperatório foi de 439,3ml no grupo RIV e 477,5ml no grupo ENO. Não houve necessidade de transfusão em nenhum paciente durante o transoperatório (tabela 6).

TABELA 6

Variáveis relacionadas ao procedimento cirúrgico

\begin{tabular}{llcc}
\hline & & $\begin{array}{c}\text { Grupo } \\
\text { rivaroxaban } \\
(\mathbf{n}=\mathbf{3 3 )} \\
\mathbf{n}(\%)\end{array}$ & $\begin{array}{c}\text { Grupo } \\
\text { enoxaparina } \\
(\mathbf{n}=\mathbf{3 2}) \\
\mathbf{n}(\%)\end{array}$ \\
\hline Lado operado & Direito & $19(58)$ & $13(41)$ \\
& Esquerdo & $14(42)$ & $19(59)$ \\
Tipo de prótese & Cimentada & $23(70)$ & $23(72)$ \\
& Não cimentada & $9(27)$ & $1(3)$ \\
Tipo de anestesia & Híbrida & $1(3)$ & $8(25)$ \\
& Raquidiana & $21(64)$ & $18(56)$ \\
Via de acesso & Aeral & $12(36)$ & $14(44)$ \\
& Antero-lateral & $27(82)$ & $23(72)$ \\
& Posterior & $6(18)$ & $9(28)$ \\
\hline Tempo de cirurgia (minutos) & $91,8(23,1)$ & $102,9(27,61)$ \\
Perda sanguínea (ml) & $439,3(141,2)$ & $477,5(249,5)$ \\
\hline
\end{tabular}

Fonte: CEDOP - Complexo Hospitalar Santa Casa de Porto Alegre

* Análise por teste do qui-quadrado, valor $p<0,05$

Todos os pacientes ficaram internados, até o quinto dia pós-operatório, dia 6 do estudo, quando foi coletada nova amostra de sangue para análise. Durante a internação receberam visitas diárias dos investigadores, nas quais foram avaliados sinais e sintomas de TVP/ TEP (calor, dor, edema, rigidez ou empastamento da musculatura da panturrilha), sangramento ativo (pela ferida operatória e pela drenagem tipo "porto vac"), e quaisquer outros eventos adversos que viessem a interferir no tratamento em estudo (infecção, reações à medicação, descompensação de comorbidades, etc.).

$\mathrm{Na}$ alta hospitalar, os pacientes recebiam orientações quanto aos cuidados no pós-operatório, juntamente com o kit contendo as drogas, para seguimento do tratamento domiciliar.

No dia 13, os pacientes retornaram ao ambulatório do hospital para retirada de pontos. Durante a consul- ta, foi avaliada a ferida operatória quanto a sinais flogísticos e presença de sangramento ativo, sinais clínicos de TVP/TEP, e encaminhados à fisioterapia para treino de marcha.

Entre os dias 32 e 36, os pacientes retornaram ao hospital para realização de flebografia bilateral com contraste em membros inferiores, no Serviço de Radiologia do Complexo Hospitalar Santa Casa de Porto Alegre/RS. No dia 36 e 65 foram coletadas novas amostras de sangue para realização dos exames laboratoriais de controle.

\section{RESULTADOS}

Neste estudo, cinco pacientes desenvolveram trombose venosa profunda no pós-operatório da artroplastia total do quadril; três pertenciam ao grupo RIV e dois, ao grupo ENO. Nenhum caso de tromboembolia pulmonar foi diagnosticado. Todos os casos de TVP foram detectados por meio da flebografia entre o $32 \mathrm{o}$ e 36을 dia pós-operatório; nenhum paciente desenvolveu sintomas clínicos sugestivos de TVP (tabela 7).

TABELA 7

Valores de ocorrência de TVP

\begin{tabular}{|c|c|c|c|}
\hline & & $\begin{array}{c}\text { Grupo } \\
\text { rivaroxaban } \\
(\mathbf{n}=33) \\
n(\%)\end{array}$ & $\begin{array}{c}\text { Grupo } \\
\text { enoxaparina } \\
(n=32) \\
n(\%)\end{array}$ \\
\hline Flebografia & $\begin{array}{l}\text { TVP + } \\
\text { TVP - }\end{array}$ & $\begin{array}{rr}3 & (9) \\
30 & (91)\end{array}$ & $\begin{array}{rr}2 & (6) \\
30 & (94)\end{array}$ \\
\hline Lado afetado pela TVP & $\begin{array}{l}\text { Ipsilateral } \\
\text { Contralateral }\end{array}$ & $\begin{array}{c}3(9) \\
-\end{array}$ & 2 (6) \\
\hline Sintomatologia & $\begin{array}{l}\text { Ausente } \\
\text { Presente }\end{array}$ & $\begin{array}{c}33(100) \\
-\end{array}$ & $\begin{array}{c}32(100) \\
-\end{array}$ \\
\hline
\end{tabular}

Fonte: CEDOP - Complexo Hospitalar Santa Casa de Porto Alegre

* Análise por teste do qui-quadrado, valor $p<0,05$

Os exames laboratoriais realizados ao longo do estudo não demonstraram diferenças estatísticas ( $\mathrm{p}<$ $0,05)$ nos itens estudados para avaliar segurança. A perda sanguínea foi avaliada por meio da dosagem da hemoglobina e do hematócrito nos dias 0, 1, 6, 13 e 36 e da perda sanguínea transoperatória e pela drenagem sanguínea pós-operatória medida pelo "porto vac". Já a toxicidade hepática foi avaliada pela dosagem das enzimas hepáticas (tabela 8). 
TABELA 8

Análise descritiva da evolução dos casos

\begin{tabular}{|c|c|c|c|c|c|}
\hline \multirow[b]{2}{*}{$\mathrm{Hb}(\mathrm{g} / \mathrm{dL})$} & \multirow[b]{2}{*}{$\begin{array}{r}6 \circ \mathrm{PO} \\
13 \circ \mathrm{PO} \\
36 \circ \mathrm{PO}\end{array}$} & \multirow{2}{*}{$\begin{array}{c}\begin{array}{c}\text { Grupo } \\
\text { rivaroxaban } \\
\text { (n }=\mathbf{3 3})\end{array} \\
\begin{aligned} \mathbf{X} & \text { (DP) }\end{aligned} \\
9,7 \quad(1,6) \\
11,0 \quad(1,2) \\
12,9 \quad(2,8)\end{array}$} & \multicolumn{2}{|c|}{$\begin{array}{c}\text { Grupo } \\
\text { enoxaparina } \\
(n=32) \\
\bar{X}(D P)\end{array}$} & \multirow{2}{*}{$\begin{array}{c}\begin{array}{c}\text { Valor } \\
\text { de } p\end{array} \\
\\
0,059 \\
0,277 \\
0,327\end{array}$} \\
\hline & & & $\begin{array}{l}10,1 \\
11,0 \\
12,6\end{array}$ & $\begin{array}{l}(1,4) \\
(1,5) \\
(1,4)\end{array}$ & \\
\hline $\mathrm{Ht}(\%)$ & $\begin{array}{r}6 \circ \mathrm{PO} \\
13 \circ \mathrm{PO} \\
36 \circ \mathrm{PO}\end{array}$ & $\begin{array}{ll}28,1 & (4,6) \\
31,7 & (4,3) \\
36,4 & (4,4)\end{array}$ & & & $\begin{array}{l}0,149 \\
0,278 \\
0,367\end{array}$ \\
\hline TGO (U/L) & $\begin{array}{r}\text { 6ㅇ } \mathrm{PO} \\
13 \circ \mathrm{PO} \\
36 \circ \mathrm{PO} \\
65 \circ \mathrm{PO}\end{array}$ & $\begin{array}{l}19,0(28,6) \\
18,5 \quad(11,8) \\
15,0(11,7) \\
17,5 \quad(9,8)\end{array}$ & $\begin{array}{l}31,4 \\
24,1 \\
22,7 \\
21,4\end{array}$ & $\begin{array}{r}(15,2) \\
(9,9) \\
(13,5) \\
(16,7)\end{array}$ & $\begin{array}{l}0,214 \\
0,278\end{array}$ \\
\hline TGP (U/L) & $\begin{array}{r}\text { 6o } \mathrm{PO} \\
13 \circ \mathrm{PO} \\
36 \circ \mathrm{PO} \\
65 \circ \mathrm{PO}\end{array}$ & $\begin{array}{l}17,0(23,6) \\
16,5(19,3) \\
18,5(10,3) \\
27,0(18,1)\end{array}$ & $\begin{array}{l}19,4 \\
24,7 \\
20,8 \\
23,9\end{array}$ & $\begin{array}{l}(17,8) \\
(18,1) \\
(12,4) \\
(25,9)\end{array}$ & $\begin{array}{l}0,201 \\
\end{array}$ \\
\hline GGT (U/L) & $\begin{array}{r}\text { 6o } \mathrm{PO} \\
13 \circ \mathrm{PO} \\
36 \circ \mathrm{PO} \\
65 \circ \mathrm{PO}\end{array}$ & $\begin{array}{l}71,5(89,2) \\
62,5(85,2) \\
97,5(42,4) \\
55,5(47,3)\end{array}$ & $\begin{array}{l}83,1 \\
81,7 \\
38,1 \\
35,0\end{array}$ & $\begin{array}{r}(102,9) \\
(85,7) \\
(31,2) \\
(29,2)\end{array}$ & $\begin{array}{l}0,313 \\
0,241 \\
0,445 \\
0,245\end{array}$ \\
\hline $\mathrm{FA}(\mathrm{U} / \mathrm{L})$ & $\begin{array}{r}\text { 6 } \mathrm{PO} \\
13 \circ \mathrm{PO} \\
36 \circ \mathrm{PO} \\
65 \circ \mathrm{PO}\end{array}$ & $\begin{array}{l}111,5(43,1) \\
111,5(23,3) \\
122,0(38,1) \\
117,0 \quad(8,4)\end{array}$ & $\begin{array}{l}80,2 \\
95,7 \\
85,6 \\
90,4\end{array}$ & $\begin{array}{l}(41,1) \\
(36,2) \\
(22,9) \\
(29,2)\end{array}$ & $\begin{array}{l}0,491 \\
0,351 \\
0,175 \\
0,386\end{array}$ \\
\hline
\end{tabular}

Fonte: CEDOP - Complexo Hospitalar Santa Casa de Porto Alegre

\section{DISCUSSÃO}

A artroplastia total de quadril é procedimento ortopédico de grande porte e, na maioria das vezes, eletivo, exceto quando por fratura do colo do fêmur. Não sendo um procedimento de urgência, é imperativa a redução máxima do risco de complicações às quais os pacientes estão sujeitos. É sabido que os pacientes submetidos a essa cirurgia apresentam risco aumentado para muitas complicações locais e sistêmicas ${ }^{(1,8)}$. Os fenômenos tromboembólicos representam os eventos adversos sistêmicos mais comuns de ATQ e são a maior causa de morte nos três primeiros meses após a operação, respondendo por mais de $50 \%$ da mortalidade pósoperatória $^{(1-2,5-10)}$. A TVP é responsável por alto número de mortes súbitas que ocorrem no pós-operatório imediato devido a embolia pulmonar, que na maioria das vezes não é diagnosticada, além de graves lesões que podem manifestar-se tardiamente. É a principal causa de morbidade e de mortalidade nas fraturas do colo do fêmur. Estudos demonstram que 10\% dos pacientes com esse tipo de fratura morrem no primeiro mês e $25 \%$, após um ano ${ }^{(2-7)}$. De acordo com o estudo de Cassone et al, a metade das mortes que ocorre no pós-operatório imediato de ATQ resulta de complicações tromboembólicas, principalmente embolia pulmonar e infarto do miocárdio; acidentes não fatais ocorrem em torno de 5 a $10 \%$ dos pacientes; e um terço dos pacientes desenvolve trombose venosa profunda ${ }^{(3)}$.

A síndrome da TVP aparece com maior frequiência nos primeiros dias após o ato cirúrgico. A ocorrência de TVP aumenta até o quarto dia pós-operatório, depois vai diminuindo; a partir do nono dia, o risco é mínimo. Mesmo assim, têm sido observados eventos de TVP, embora pouco freqüentes, até o 17o dia pósoperatório $^{(2,4-5,11-12)}$. A incidência de TEP na prótese total do quadril oscila em torno de $6 \%$ e pode surgir até a quarta semana ${ }^{(3,8)}$. Fredin et al verificaram que o risco máximo de desenvolvimento de embolia pulmonar fatal ocorreu durante a segunda e a terceira semanas de pós-operatório ${ }^{(12)}$. Sem a adequada profilaxia, a TVP pode ocorrer em $40 \%$ a $70 \%$ dos pacientes submetidos à $\mathrm{ATQ}^{(1-7,9-13)}$. Ela evolui para embolia pulmonar fatal em $0,5 \%$ a $2 \%$ desses pacientes ${ }^{(2,8-10)}$. Sabe-se que a incidência de TVP e de TEP aumenta com a idade. Nos pacientes da faixa etária de 60 anos, a incidência varia de 30 a $35 \%$, enquanto nos acima de 70 anos é de 50 a $70 \%$, mesmo quando foram submetidos a algum tipo de profilaxia ${ }^{(2-3,7,13)}$. Em nosso estudo, a média de idade dos pacientes que desenvolveram TVP foi de 59,2 anos; o paciente mais novo tinha apenas 30 anos.

A formação de trombose está associada à presença dos fenômenos conhecidos como tríade de Virchow: estase venosa, lesão endotelial e hipercoagulabilidade $^{(4)}$. Cerca de $20 \%$ a $30 \%$ dos coágulos podem ter origem primária no segmento venoso iliofemoral, sem relação com trombos provenientes da panturrilha. Aproximadamente $80 \%$ a $90 \%$ das tromboses ocorrem no membro operado ${ }^{(2-3,11-12)}$. Em sua maioria, os coágulos da panturrilha são pequenos e clinicamente insignificantes. Segundo Cassone et al, o diagnóstico de TVP baseado apenas na sintomatologia é pouco preciso, tendo alta incidência de falso-positivos e falso- 
negativos $^{(3)}$. Há falha diagnóstica em $50 \%$ a $90 \%$ dos exames clínicos e é feito diagnóstico falso-positivo de TVP em $50 \%$ das suspeitas clínicas ${ }^{(9-10)}$.

Em nosso estudo, em todos os pacientes que apresentaram TVP, ela ocorreu no lado operado; a origem do trombo foi em $80 \%$ dos casos no segmento iliofemoral e apenas $20 \%$ nas veias profundas da panturrilha. Corroborando os achados de Cassone et $a^{(3)}$, nenhum dos pacientes com diagnóstico de TVP apresentou sintomatologia clínica.

O estudo de Albuquerque et al, relação entre o tempo decorrido a partir da operação e o início do desenvolvimento das complicações tromboembólicas: $29 \%$ dos trombos desenvolvem-se nos primeiros 12 dias após a cirurgia e $23 \%$ entre o $12^{\circ}$ e o $24^{\circ}$ dias; o risco máximo de desenvolvimento de embolia pulmonar fatal ocorreu durante a segunda e a terceira semanas de pós-operatório ${ }^{(2)}$.

Vários fatores relacionados à técnica cirúrgica de ATQ estão associados ao desenvolvimento da TVP/TEP ${ }^{(1-}$ ${ }^{3,13)}$. Estase venosa pode ocorrer como resultado do posicionamento do membro durante o procedimento, do edema pós-operatório localizado, da mobilidade reduzida após a cirurgia e da redução substancial do fluxo venoso dos membros inferiores no transoperatório $^{(1-3,14)}$. A lesão do endotélio pode ocorrer como resultado do posicionamento e da manipulação do membro inferior na mesa cirúrgica ou da reação térmica causada pela cimentação dos componentes da próte$\mathrm{se}^{(13)}$. A hipercoagulabilidade pode resultar do trauma associado à ATQ que ativa os fatores trombogênicos que apresentarão tropismo por áreas de lesão vascular e estase venosa ${ }^{(5,11,13)}$. A perda sanguínea associada ao procedimento cirúrgico pode reduzir os níveis de antitrombina III e inibir o sistema fibrinolítico endógeno, permitindo, assim, a formação e o crescimento do trombo, que ocorre em vasos da pelve, coxa e panturrilha ${ }^{(3,11-}$ 12).

São fatores de risco para o desenvolvimento de TVP: indivíduos com idade superior a 40 anos (incidência aumenta com a idade), obesidade (IMC $>30 \mathrm{~kg} / \mathrm{m}^{2}$ ), varizes e cirurgias venosas e ortopédicas prévias, história de tromboembolismo (caráter recorrente), ocorrência em membros da família (hereditariedade), por- tadores de anormalidade genética do sistema de coagulação, uso de anticoncepcionais orais (sobretudo em mulheres fumantes), terapia de reposição hormonal, câncer e quimioterapia, gravidez, puerpério e período pós-parto, doenças respiratórias ou cardíacas graves, infecções graves, traumatismos, cirurgias de grande porte, anestesia de longa duração, período pós-operatório, perda excessiva de sangue, transfusão, hospitalização prolongada, imobilização no leito, viagens de longa duração (síndrome da classe turística) ${ }^{(2-3,11-13)}$.

Devido ao rigor da seleção dos pacientes em nosso estudo, muitos dos fatores de risco para o desenvolvimento de TVP foram eliminados por enquadrarem-se nos critérios de exclusão. Dentre os pacientes do estudo com diagnóstico de TVP por flebografia, $80 \%$ tinham mais de 40 anos, nenhum era tabagista ou obeso e apenas um apresentava varizes em membros inferiores. Em todas essas cirurgias foi utilizada a via de acesso ântero-lateral e a prótese foi cimentada. Os níveis de hemoglobina de $80 \%$ dos pacientes estavam abaixo do normal; $60 \%$ dos pacientes com diagnósticos de TVP apresentaram queda de $2 \mathrm{~g} / \mathrm{dl}$ ou mais nos níveis de hemoglobina no pós-operatório; contudo, nenhum paciente com flebografia positiva para TVP recebeu transfusão sanguínea no transoperatório ou pós-operatório. Devido ao grande número de fatores de risco existentes para o desenvolvimento de TVP, sua profilaxia torna-se extremamente importante ${ }^{(15-21)}$.

Atualmente, utilizam-se para esses fins drogas como a heparina não fracionada, a heparina de baixo peso molecular (como a enoxaparina) ${ }^{(22-24)}$ e os antagonistas da vitamina $\mathrm{K}^{(9-10,25-31)}$. Apesar de esses fármacos serem eficazes em diminuir a incidência de TVP e TEP após cirurgia ortopédica, certas limitações restringem seu uso. Os antagonistas da vitamina $\mathrm{K}$, apesar de administrados por via oral, apresentam início de ação lento, alta variabilidade entre a resposta dos pacientes à droga, necessidade de monitorização laboratorial freqüente, para evitar superdosagens, além de potencial interação com outras drogas ${ }^{(2,18-19)}$. Por sua vez, ambas as heparinas necessitam ser administradas por via parenteral, o que poderia ser considerado um fator para diminuir a adesão ao tratamento, além do desconforto da via $^{(4,14,28-33)}$. 
O rivaroxaban é um anticoagulante oral que age inibindo diretamente a ação do fator de coagulação $X$ ativado $(\mathrm{FXa})^{(14,34-38)}$. Sua concentração plasmática máxima é atingida duas a quatro horas após a administração oral e sua biodisponibilidade varia de 60 a $80 \%{ }^{(34,39)}$. Ele apresenta rápido início de ação e sua farmacocinética e farmacodinâmica são previsíveis ${ }^{(36)}$. O rivaroxaban é excretado por via renal (66\% da dose total) e biliar (28\%); 36\% são excretados inalterados na urina ${ }^{(14,34,36,39)}$. Além disso, por apresentar ampla janela terapêutica, não necessita monitorização laboratorial de rotina e pode ser administrado em dose úni$\mathrm{ca}^{(14,35-38,40-42)}$.

Este trabalho tem como objetivo comparar a eficácia e a segurança de rivaroxaban e da enoxaparina na profilaxia de TVP após ATQ. Não houve redução estatisticamente significativa $(\mathrm{p}<0,05)$ na prevenção de TVP quando comparadas as duas drogas.

A segurança do tratamento em estudo foi analisada por meio da perda sanguínea, que foi avaliada através dos seguintes critérios: a) queda de $2 \mathrm{~g} / \mathrm{dl}$ ou mais dos níveis de hemoglobina $(\mathrm{Hb})$ do pré-operatório em relação ao pós ${ }^{(15-16)}$; b) necessidade de duas ou mais unidades de concentrado de hemácias $(\mathrm{CHAD})^{(43-46)}$. No grupo RIV, 14 pacientes necessitaram de transfusão de duas ou mais unidades de CHAD no pós-operatório; no grupo ENO, 12 pacientes necessitaram de transfusão de duas ou mais unidades de CHAD. O grupo RIV apresentou diminuição média na $\mathrm{Hb}$ de $1 \mathrm{~g} / \mathrm{dl}$ durante o seguimento do tratamento e o grupo ENO, queda de $0,8 \mathrm{~g} / \mathrm{dl}$, o que nos permite verificar que as medicações são semelhantes no que se refere à segurança do tratamento $(\mathrm{p}<0,05)$. Eriksson et al observaram, em estudo comparativo entre rivaroxaban e enoxaparina, que as taxas de sangramento clinicamente relevantes durante o período de tratamento com tais drogas foram menores no grupo rivaroxaban; entretanto, as taxas de transfusão sanguínea e o volume transfundido mostraram-se similares entre os dois grupos ${ }^{(14)}$.

Foram verificadas alterações nos valores laboratoriais das enzimas hepáticas em ambos os grupos. A incidência de elevação nos valores de ALT no grupo RIV ficou em torno de 3\% e, no grupo ENO, 10\%; a elevação verificada não foi dose-dependente. Os valo- res de AST também apresentaram-se alterados em ambos os grupos, com elevação em torno de $6 \%$ no grupo RIV e $10 \%$ no grupo ENO. As alterações foram verificadas a partir do 7o dia de administração das medicações e não houve repercussão clínica sugestiva de prejuízo da função hepática em nenhum dos pacientes que apresentaram tais alterações ${ }^{(14,35)}$.

Segundo os estudos de Eriksson et al, o rivaroxaban apresentou, na fase final de testes, taxas de ocorrência de TVP/TEP de 1,1\%, que, em comparação com as taxas de $3,7 \%$ apresentadas pelo grupo em uso de enoxaparina, evidenciaram redução relativa de risco de $88 \%(\mathrm{p}<0,001)$ na ocorrência dos eventos tromboembólicos ${ }^{(24)}$. Devido à facilidade na posologia e ao rápido inicio de ação, o rivaroxaban - e só devido a essas características - pode vir a ser considerado excelente substituto da enoxaparina na prevenção de eventos tromboembólicos nas cirurgias ortopédicas de grande porte.

\section{CONCLUSÃO}

Após a realização deste estudo, duplo-cego, randomizado e comparativo entre rivaroxaban (RIV) e enoxaparina (ENO), para avaliação de eficácia e segurança na profilaxia da trombose venosa profunda e do tromboembolismo pulmonar em 65 pacientes submetidos à artroplastia total do quadril, concluímos que não houve diferença estatisticamente significante $(\mathrm{p}<$ $0,05)$, quando comparadas eficácia e segurança entre os fármacos rivaroxaban e enoxaparina.

\section{REFERÊNCIAS}

1. Huo MH, Gilbert NF, Parvizi J. What's new in total hip arthroplasty. J Bone Joint Surg Am. 2007;89(8):1874-85. Review.

2. Albuquerque HPC, Vidal PC. Trombose venosa profunda: revisão dos conceitos atuais. Rev Bras Ortop. 1996;31(10): 851-6.

3. Cassone A, Viegas AC, Sguizzatto GT, Cabrita HABA, Aquino MA, Furlaneto ME, et al. Trombose venosa profunda em artroplastia total de quadril. Rev Bras Ortop. 2002;37(5):153-61.

4. Schwartsmann CR, Cavalieri Costa R, Drumand SN, Maciel AC, Molina MAP, Garzella MM, Damin M. Estudo aberto, randomizado, comparativo, para avaliar a eficácia e segurança da enoxaparina comparada à heparina não fracionada na profilaxia do trombembolismo venoso em pacientes submetidos a artroplastia total do quadril. Rev Bras Ortop. 1996;31(10):797-808. 
5. O'Reilly RF, Burgess IA, Zicat B. The prevalence of venous thromboembolism after hip and knee replacement surgery. Med J Aust. 2005;182(4):154-9. Comment in: Med J Aust. 2005; 182(4):149-50.

6. Geerts WH, Pineo GF, Heit JA, Bergqvist D, Lassen MR, Colwell CW, Ray JG. Prevention of venous thromboembolism: the Seventh ACCP Conference on Antithrombotic and Thrombolytic Therapy. Chest. 2004;126(3 Suppl):338S-400S. Comment in: Chest. 2005;127(6):2297-8.

7. Leizorovicz A; SMART Vennography Study Steering Commitee. Epidemiology of post-operative venous thromboembolism in Asian patients. Results of the SMART venography study. Haematologica. 2007;92(9):1194-200.

8. Bowler DJ, Bale E, O'Byrne J. Factor V Leiden: prevalence and thromboembolic complications after total hip replacement in Ireland. Ir J Med Sci. 2007;176(4):273-7.

9. Strebel N, Prins M, Agnelli G, Büller HR. Preoperative or postoperative start of prophylaxis for venous thromboembolism with low-molecular-weight heparin in elective hip surgery? Arch Intern Med. 2002;162(13):1451-6. Comment in: Arch Intern Med. 2003;163(3):369; author reply 369.

10. Prandoni P, Bruchi O, Sabbion P, Tanduo C, Scudeller A, Sardella C, et al. Prolonged thromboprophylaxis with oral anticoagulants after total hip arthroplasty: a prospective controlled randomized study. Arch Intern Med. 2002;162(17): 1966-71.

11. Sharrock NE, Go G, Harpel PC, Ranawat CS, Sculco TP, Salvati EA. The John Charnley Award. Thrombogenesis during total hip arthroplasty. Clin Orthop Relat Res. 1995;(319):1627.

12. Fredin H, Nilsson B, Rosberg B, Tengborn L. Pre- and postoperative levels of antithrombin III with special reference to thromboembolism after total hip replacement. Thromb Haemost. 1983;49(3):158-61.

13. Schiff RL, Kahn SR, Shrier I, Strulovitch C, Hammouda W, Cohen E, Zukor D. Identifying orthopedic patients at high risk for venous thromboembolism despite thromboprophylaxis. Chest. 2005;128(5):3364-71.

14. Eriksson BI, Borris LC, Dahl OE, Haas S, Huisman MV, Kakkar K, Muehlhofer E, Dierig C, Misselwitz F, Kälebo P; ODIXa-HIP Study Investigators. A once-daily, oral, direct Factor Xa inhibitor, rivaroxaban (BAY 59-7939), for thromboprophylaxis after total hip replacement. Circulation. 2006;114(22):2374-81. Comment in: Circulation. 2006; 114(22):2313-6.

15. Johansson T, Engquist M, Pettersson LG, Lisander B. Blood loss after total hip replacement: a prospective randomized study between wound compression and drainage. J Arthroplasty. 2005;20(8):967-71.

16. Wallis JP, Wells AW, Whitehead S, Brewster N. Recovery from post-operative anaemia. Transfus Med. 2005;15(5):413-8.

17. Fuchs S, Heyse T, Rudofsky G, Gosheger G, Chylarecki C. Continuous passive motion in the prevention of deep-vein thrombosis: a randomised comparison in trauma patients. J Bone Joint Surg Br. 2005;87(8):1117-22.
18. Stannard JP, Lopez-Ben RR, Volgas DA, Anderson ER, Busbee M, Karr DK, et al. Prophylaxis against deep-vein thrombosis following trauma: a prospective, randomized comparison of mechanical and pharmacologic prophylaxis. J Bone Joint Surg Am. 2006;88(2):261-6.

19. Friedman RJ, Gallus AS, Cushner FD, Fitzgerald G, Anderson FA Jr; Global Orthopaedic Registry Investigators. Physician compliance with guidelines for deep-vein thrombosis prevention in total hip and knee arthroplasty. Curr Med Res Opin. 2008;24(1):87-97.

20. McNally MA. Insufficient duration of venous thromboembolism prophylaxis after total hip or knee replacement when compared with the time course of thromboembolic events. J Bone Joint Surg Br. 2007;89(10): 1409; author reply 1409-10.

21. Eriksson BI, Quinlan DJ. Oral anticoagulants in development: focus on thromboprophylaxis in patients undergoing orthopaedic surgery. Drugs. 2006;66(11):1411-29.

22. Leclerc JR, Gent M, Hirsh J, Geerts WH, Ginsberg JS. The Incidence of symptomatic venous thromboembolism during and after prophylaxis with enoxaparin: a multi-institutional cohort study of patients who underwent hip or knee arthroplasty. Canadian Collaborative Group. Arch Intern Med. 1998;158(8):873-8.

23. Eriksson BI, Dahl OE, Rosencher N, Kurth AA, van Dijk CN, Frostick SP, Prins MH, Hettiarachchi R, Hantel S, Schnee J, Büller HR; RE-NOVATE Study Group. Dabigatran etexilate versus enoxaparin for prevention of venous thromboembolism after total hip replacement: a randomised, double-blind, noninferiority trial. Lancet. 2007;370(9591): 949-56. Erratum in: Lancet. 2007;370(9604):2004. Comment in: Lancet. 2007;370 (9604):2002-3; author reply 2003-4. Lancet. 2007;370(9604) 2002; author reply 2003-4. Lancet. 2007;370(9591):915-7.

24. Eriksson BI, Borris LC, Friedman RJ, Haas S, Huisman MV, Kakkar AK, Bandel TJ, Beckmann H, Muehlhofer E, Misselwitz F, Geerts W; RECORD1 Study Group. Rivaroxaban versus enoxaparin for thromboprophylaxis after hip arthroplasty. N Engl J Med. 2008;358(26):2765-75. Comment in: N Engl J Med. 2008;358(26):2827-9.

25. Bergqvist D, Benoni G, Björgell O, Fredin H, Hedlundh U, Nicolas S, et al. Low-molecular weight heparin (enoxaparin) as prophylaxis against venous thromboembolism after total hip replacement. N Engl J Med. 1996;335(10):696-700. Comment in: ACP J Club. 1997;126(1):11. N Engl J Med. 1997;336(8): 585; author reply 586. N Engl J Med. 1997;336(8):585; author reply 586

26. Hirsh J, O’Donnell M, Eikelboom JW. Beyond unfractionated heparin e warfarin: current and future advances. Circulation. 2007;116(5):552-60. Review.

27. Wannmacher L. Heparinas de baixo peso molecular: evidências que fundamentam indicações. Uso Racional de Medicamentos: Temas Selecionados. 2007;4(2):1-6.

28. Holzheimer RG. Low-molecular-weight heparin (LMWH) in the treatment of thrombosis. Eur J Med Res. 2004;9(4):225-39. Review.

29. Noble SI, Nelson A, Turner C, Finlay IG. Acceptability of low molecular weight heparin thromboprophylaxis for inpatients 
receiving palliative care: qualitative study. BMJ. 2006;332 (7541):577-80. Comment in: BMJ. 2006;332(7543):728-9. BMJ. 2006;332(7543):729.

30. Hull RD, Pineo GF, Brant RF, Mah AF, Burke N, Dear R, Wong T, Cook R, Solymoss S, Poon MC, Raskob G; LITE Trial Investigators. Self-managed long-term low-molecular-weight heparin therapy: the balance of benefits and harms. Am J Med. 2007;120(1):72-82.

31. Kearon C, Ginsberg JS, Julian JA, Douketis J, Solymoss S, Ockelford P, Jackson S, Turpie AG, MacKinnon B, Hirsh J, Gent M; Fixed-Dose Heparin (FIDO) Investigators. Comparison of fixed-dose weight-adjusted unfractionated heparin and low-molecular-weight heparin for acute treatment of venous thromboembolism. JAMA. 2006;296(8):935-42.

32. Cardiovascular Disease Educational and Research Trust; Cyprus Cardiovascular Disease Educational and Research Trust; European Venous Forum; International Surgical Thrombosis Forum; International Union of Angiology; Union Internationale de Phébologie. Prevention and treatment of venous thromboembolism. International Consensus Statement (guidelines according to scientific evidence). Int Angiol. 2006; 25(2):101-61.

33. Tapson VF, Hyers TM, Waldo AL, Ballard DJ, Becker RC, Caprini JA, Khetan R, Wittkowsky AK, Colgan KJ, Shillington AC; NABOR (National Anticoagulation Benchmark and Outcomes Report) Steering Committee. Antithrombotic therapy practices in US hospitals in an era of practice guidelines. Arch Intern Med. 2005;165(13):1458-64. Comment in: Arch Intern Med. 2005;165(13):1455-6. Arch Intern Med. 2006;166(1):126.

34. Turpie AG, Gallus AS, Hoek JA;Pentasaccharide Investigators. A synthetic pentasaccharide for the prevention of deep-vein thrombosis after total hip replacement. N Engl J Med. 2001; 344(9):619-25. Comment in: N Engl J Med. 2001;345(4):2912. N Engl J Med. 2001;345(4):291; author reply 292.

35. Agnelli G, Gallus A, Goldhaber SZ, Haas S, Huisman MV, Hull RD, Kakkar AK, Misselwitz F, Schellong S; ODIXa-DVT Study Investigators. Treatment of proximal deep-vein thrombosis with the oral direct factor Xa inhibitor rivaroxaban (BAY 59-7939): the ODIXa-DVT (Oral Direct Factor Xa Inhibitor BAY 59-7939 in Patients With Acute Symptomatic Deep-Vein Thrombosis) study. Circulation. 2007;116(2):1807. Comment in: Circulation. 2007;116(2):131-3.

36. Kubitza D, Becka M, Voith B, Zuehlsdorf M, Wensing G. Safety, pharmacodynamics, and pharmacokinetics of single doses of BAY 59-7939, an oral, direct factor Xa inhibitor. Clin Pharmacol Ther. 2005;78(4):412-21.
37. Mueck W, Eriksson BI, Bauer KA, Borris L, Dahl OE, Fisher WD, et al. Population pharmacokinetics and pharmacodynamics of rivaroxaban - an oral, direct factor Xa inhibitor - in patients undergoing major orthopaedic surgery. Clin Pharmacokinet. 2008;47(3):203-16.

38. Eriksson BI, Borris L, Dahl OE, Haas S, Huisman MV, Kakkar AK, Misselwitz F, Kälebo P; ODIXa-HIP Study Investigators. Oral, direct Factor Xa inhibition with BAY 59-7939 for the prevention of venous thromboembolism after total hip replacement. J Thromb Haemost. 2006;4(1):121-8.

39. Turpie AG, Bauer KA, Eriksson BI, Lassen MR. Fondaparinux vs enoxaparin for the prevention of venous thromboembolism in major orthopedic surgery: a meta-analysis of 4 randomized double-blind studies. Arch Intern Med. 2002;162(16):1833-40. Comment in: Arch Intern Med. 2002;162(16):1806-8. Arch Intern Med. 2003;163(22):2794; author reply 2794-5.

40. Graff J, von Hentig N, Misselwitz F, Kubitza D, Becka M, Breddin HK, Harder S. Effects of the oral, direct factor xa inhibitor rivaroxaban on platelet-induced thrombin generation and prothrombinase activity. J Clin Pharmacol. 2007;47(11): 1398-407.

41. Laux V, Perzborn E, Kubitza D, Misselwitz F. Preclinical and clinical characteristics of rivaroxaban: a novel, oral, direct factor Xa inhibitor. Semin Thromb Hemost. 2007;33(5):51523.

42. Turpie AG, Fisher WD, Bauer KA, Kwong LM, Irwin MW, Kälebo P, Misselwitz F, Gent M; OdiXa-Knee Study Group. BAY 59-7939: an oral, direct factor $\mathrm{Xa}$ inhibitor for the prevention of venous thromboembolism in patients after total knee replacement. A phase II dose-ranging study. J Thromb Haemost. 2005;3(11):2479-86.

43. Ho KM, Ismail H. Use of intravenous tranexamic acid to reduce allogeneic blood transfusion in total hip and knee arthroplasty: a meta-analysis. Anaesth Intensive Care. 2003;31(5):529-37.

44. Weber EW, Slappendel R, Prins MH, van der Schaaf DB, Durieux ME, Strümper D. Perioperative blood transfusions and delayed wound healing after hip replacement surgery: effects on duration of hospitalization. Anesth Analg. 2005;100(5): 1416-21, table of contents. Comment in: Anesth Analg. 2005; 101(6):1889-90; author reply 1890.

45. Alencar PGC, Camargo J, Meyer AT, Ernlund L, Molinari S. Autotransfusão sanguínea em artroplastia total do quadril. Rev Bras Ortop. 1994;29(6):389-92.

46. Rollo VJ, Hozack WJ, Rothman RH, Chao W, Eng KO. Prospective randomized evaluation of blood salvage techniques for primary total hip arthroplasty. J Arthroplasty. 1995;10(4): 532-9.

\footnotetext{
Declaração de inexistência de conflitos de interesse: Pesquisadores e a instituição onde foi realizada a pesquisa receberam suporte financeiro do Laboratório Bayer Healthcare - entretanto, esse fato não implicou conflito de interesse de qualquer natureza.
} 